\title{
Scale-Invariant Feature Transform (SIFT) Algorithm-Based Detection of Cardiac Insufficiency in Sepsis Patients with Echocardiography
}

\author{
Xiongxiong Wang $\mathbb{D}^{1},{ }^{1}$ Li Xu $\mathbb{D}^{1},{ }^{1}$ Xuena Cui $\mathbb{D}^{1},{ }^{1}$ Hefei Zhu $\mathbb{D}^{1},{ }^{1}$ and Min Zhang $\mathbb{D}^{2}$ \\ ${ }^{1}$ Department of Intensive Care Medicine, YinZhou Second Hospital, Ningbo 315040, Zhejiang, China \\ ${ }^{2}$ Teaching and Research Division of Internal Medicine, Hubei College of Chinese Medicine, Jingzhou 434020, Hubei, China
}

Correspondence should be addressed to Min Zhang; 33215208@njau.edu.cn

Received 13 December 2021; Revised 20 January 2022; Accepted 22 January 2022; Published 22 February 2022

Academic Editor: M Pallikonda Rajasekaran

Copyright (c) 2022 Xiongxiong Wang et al. This is an open access article distributed under the Creative Commons Attribution License, which permits unrestricted use, distribution, and reproduction in any medium, provided the original work is properly cited.

\begin{abstract}
This research aimed to explore the application of cardiac ultrasound imaging (CUI) in the examination and diagnosis of sepsis patients with cardiac insufficiency under a speckle tracking algorithm (STA). Scale-invariant feature transform (SIFT) algorithm was introduced to process images of CUI through STA under feature points of cardiac ultrasonic images. 90 patients with sepsis who were admitted to the hospital were selected and randomly divided into a sound cardiac function group $(n=62)$ (group A) and a cardiac insufficient group $(n=28)$ (group B) under whether they had cardiac insufficiency, and 20 healthy people were selected as a control group. Sepsis patients were examined on the seventh day after diagnosis including laboratory-related indicators, echocardiography, and echocardiographic data. The results showed that there were differences in cardiac ultrasound, cardiac marker, and laboratory examinations of subjects in the three groups $(P<0.05)$. Creatine kinase isoenzyme-MB $(\mathrm{CK}-\mathrm{MB})$, glycogen phosphorylase isoenzyme BB (GPBB), and heart-type fatty acid-binding protein (H-FABP) of subjects in the three groups showed statistically marked differences $(P<0.05)$. The other results indicated tricuspid late diastolic blood flow velocity $\left(\mathrm{A}^{\prime}\right)$ value was greater than stroke volume (SV) and isovolumetric relaxation time (IVRT) value, followed by left ventricular ejection fraction (EF) and cardiac output per minute output (CO), and EF and CO values were over peak velocity of right ventricular free wall tricuspid valve systolic movement (SM) and left ventricular fractional shortening (FS) value, and left ventricular end-diastolic volume (EDV) value came next. The analysis of CUI based on STA could help doctors to judge sepsis patients with cardiac function. In addition, sepsis patients with sound cardiac function and sepsis patients with cardiac insufficiency could be distinguished by CUI, which had certain guiding significance for clinical diagnosis.
\end{abstract}

\section{Introduction}

Sepsis has attracted more and more attention because of its high mortality and morbidity [1-3]. There are often multiple organs affected by sepsis, among which the heart is one of the most seriously affected organs. When sepsis patients suffered from septic shock, $30 \%-80 \%$ of them die because of severe damage to the cardiovascular system [4]. Most patients with sepsis have varying degrees of cardiac insufficiency, which will lead to reduced perfusion of the whole body tissues to promote the development of sepsis; thus, the rest organs were further in failure $[5,6]$. At present, cardiac insufficiency is usually examined by CUI, and there are twodimensional speckle tracking imaging (2D-STI) and threedimensional STI (3D-STI) in ultrasonic examination [7]. $3 \mathrm{D}-\mathrm{STI}$ is a new technology developed in recent years, which overcomes the defects of angle dependence in Doppler, and 2D-STI is limited to defects in the plane, which makes the evaluation of left ventricular function more realistic and comprehensive. However, 3D-STI still has some dense, irregular, and speckle noise, which makes the overall image relatively fuzzy. Therefore, the algorithm is further introduced to further improve the image quality, so as to make the image clear [8]. 
Cardiac ultrasound is the best imaging detection method for the clinical assessment of cardiac structure and function of severe patients, but most patients with sepsis have already developed severe organ dysfunction when changes in cardiac structure and function are observed by CUI. In addition, echocardiographic examination is limited to the influences of equipment and the operator's clinical experience and subjective judgment, so its sensitivity is relatively poor. Thus, it is necessary to supplement the corresponding indexes for the evaluation of cardiac function $[9,10]$. In the case of cardiac insufficiency, the myocardial cells are damaged and the heart structure and function are changed so that the concentration of various cardiac markers in the blood is correspondingly increased, which can appear in the serum at the early stage of cardiac function impairment. Thus, their concentrations can be used as good early warnings and supplementary indicators for echocardiography evaluation of cardiac function $[11,12]$. What is more, SIFT has been widely applied in the processing of clinical imaging. For example, Shiji et al. [13] applied SIFT and Canny edge detection algorithm to automatically detect accurate segmentation of breast ultrasound images and found that the true-positive rate was as high as $90.1 \%$ compared to the real situation. Besides, it is of great significance to employ the intelligent algorithm in the preprocessing of clinical medical images to effectively segment the useful information in the images and promote the clinical diagnosis rate of diseases.

Therefore, CUI on the basis of STA was adopted in this study for diagnosis, so as to further study the diagnostic effects of cardiac ultrasonic images in sepsis patients with cardiac insufficiency.

\section{Materials and Methods}

2.1. Feature Point Location and Matching of SIFT. SIFT operator was a kind of image local feature description operator based on scale space and invariant image scaling, rotation, and even radiation transformation. SIFT could correspondingly combine the steps of feature point detection, feature vector generation, and feature matching search, which were optimized based on the actual situation. Besides, two images with a very $t$ large difference could also achieve feature matching. The specific process of SIFT matching is shown in Figure 1.

Accurate positioning of SIFT was often achieved by difference of Gaussian (DOG) pyramids, and the DOG value of the DOG pyramid was sensitive to noise and edge. The location and scale of feature points were adjusted accurately through further three-dimensional quadratic function fitting for local extremum points. In addition, the Taylor expansion equation of scale-space function $D(a, b, \alpha)$ in local extreme value point $D\left(a_{0}, b_{0}, \alpha_{0}\right)$ is shown as follows:

$$
D(a, b, \alpha)=D\left(a_{0}, b_{0}, \alpha_{0}\right)+\frac{\partial D^{T}}{\partial E} E+\frac{1}{2} E^{T} \frac{\partial^{2} D}{\partial E^{2}} E .
$$

In equation (1), $E=(a, b, \alpha)^{T}$ is the deviation of a key point and $D\left(a_{0}, b_{0}, \alpha_{0}\right)$ is the value of $D(a, b, \alpha)$ at the local extremum of the image. What is more, it is necessary to further take the derivative of equation (1) to obtain $\partial D(E) / E=0$, so as to get the accurate extremum location $\widehat{E}$, as shown in the following equation:

$$
\widehat{E}=-\frac{\partial^{2} D^{-1} \partial D}{\partial E^{2} \partial E} \text {. }
$$

When the deviation of $\widehat{E}$ from the interpolation center in any direction was greater than 0.5 , it meant that the key point motivated another monitoring point to be very close, and this point should be deleted.

The reference direction of local image features was obtained through the above processes. Besides, the Gaussian image closest to the scale value was gained from the obtained feature point scale value $\alpha$. The calculation method could be expressed asfollows:

$$
L(a, b, \alpha)=G(a, b, \alpha) \cdot I(a, b) .
$$

The finite difference was employed to calculate the gradient direction and gradient modulus of the image gradient in the region with the radius of $3 \times 1.5$ and the feature point as its center. Equations (4)-(6) represent the gradient $\operatorname{grad} I(a, b)$ of feature point, gradient modulus $n$, and gradient direction $\theta$, respectively.

$$
\begin{aligned}
\operatorname{gra} d I(a, b) & =\left(\frac{\partial I}{\partial a}, \frac{\partial I}{\partial b}\right), \\
n(a, b) & =\sqrt{(L(a+1, b)-L(a-1, b))^{2}+(L(a, b+1)-L(a, b-1))^{2}}, \\
\theta(a, b) & =\tan ^{-1}\left[\frac{L(a, b+1)-L(a, b-1)}{L(a+1, b)-L(a-1, b)}\right],
\end{aligned}
$$

After the gradient feature points of the Gaussian image were calculated completely, the gradient mode value and gradient direction of pixels in the neighborhood were further collected through histogram, as shown in Figure 2.
2.2. Subjects Investigated and Grouping. A total of 90 patients with sepsis were selected, who were admitted to the hospital from January 2018 to May 2020. All the selected patients did not die or were hospitalized for less than 3 days during their 


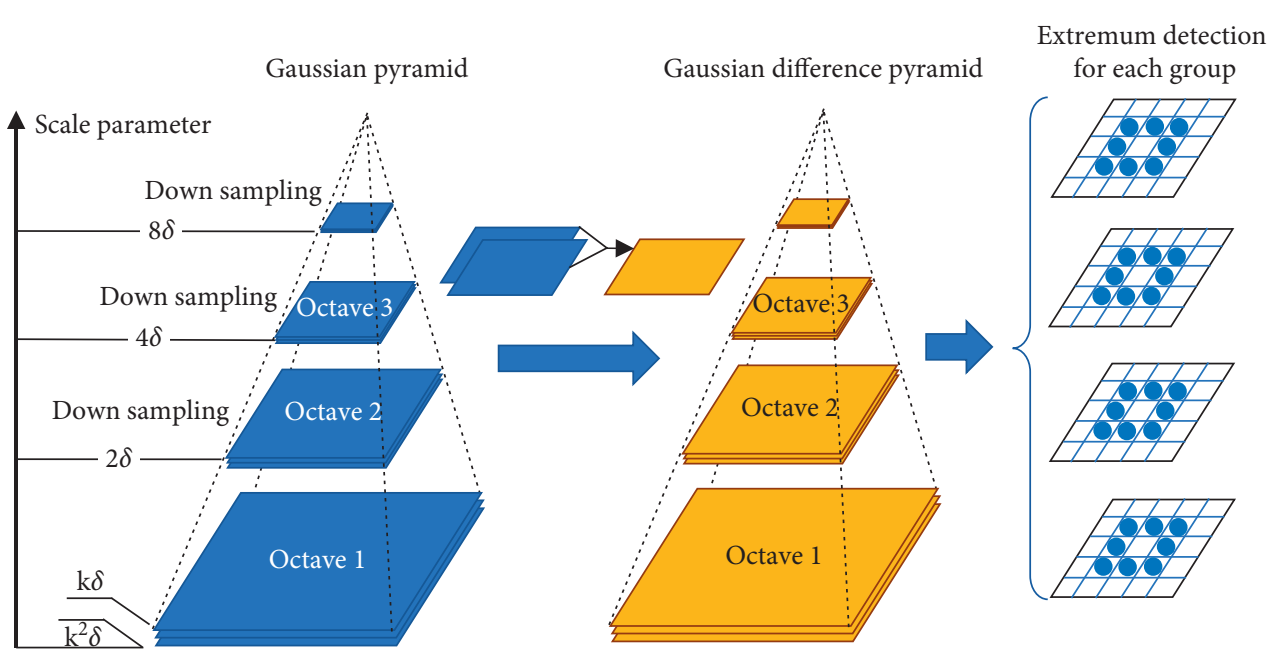

FIGURE 1: Flowchart of SIFT.

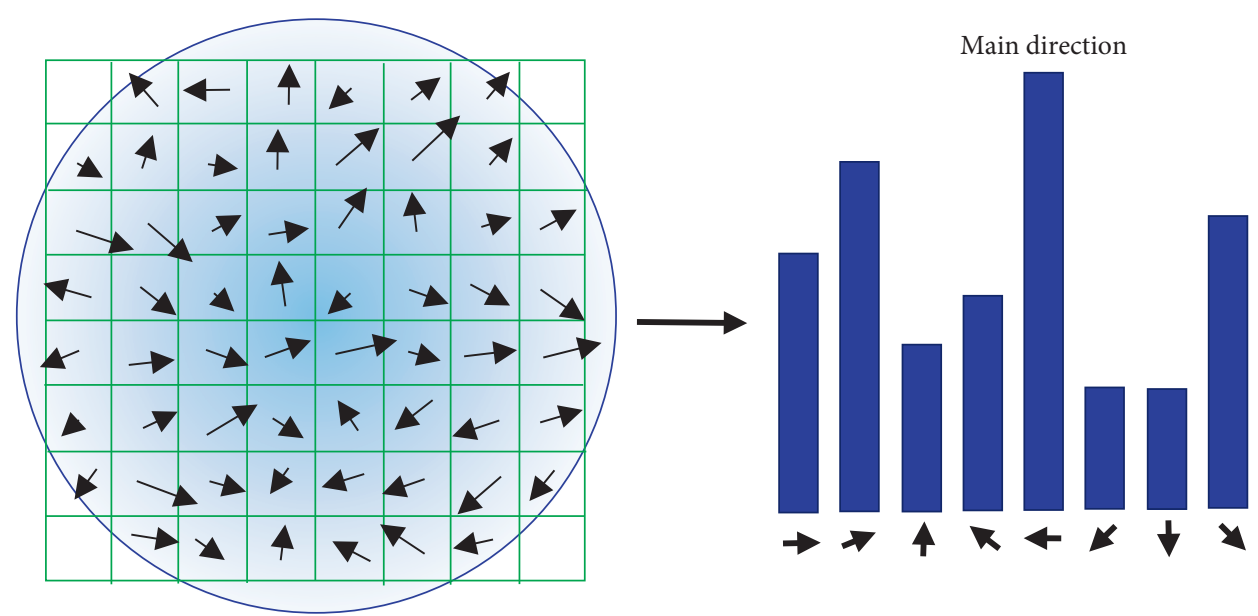

FIgURE 2: Histogram of gradient direction.

hospitalization. There were 54 males and 36 females in the experiment with an average age of $72.58 \pm 10.27$ years. The sources of sepsis infection included pulmonary infection, abdominal infection, urinary system infection, central nervous system infection, and blood infection. Moreover, 20 healthy people were selected as the control group for the study, who underwent a normal physical examination in the hospital. The patients with sepsis were divided into two groups (groups A and B) under whether they had cardiac insufficiency or not. All patients participating in the experiment had signed informed consent forms, and this study had been approved by the ethics committee of the hospital.

2.3. Inclusion and Exclusion Criteria. All the enrolled patients were diagnosed based on the diagnostic criteria of sequential organ failure assessment (SOFA), and sepsis could be confirmed when the SOFA score was greater than or equal to 2. All the SOFA scores are shown in Table 1.

The exclusion criteria were defined as follows: patients who suffered from myocardial diseases such as myocardial infarction, old myocardial infarction, and ischemic cardiomyopathy; had primary cardiomyopathy including hypertrophic cardiomyopathy, dilated cardiomyopathy, and restrictive cardiomyopathy; suffered from metabolic cardiomyopathies such as anaemic cardiomyopathy, hyperthyroidism cardiomyopathy, and uremia cardiomyopathy; had congenital heart disease; had received other treatments including traditional Chinese medicine; and refused to accept rehabilitation training with poor compliance.

2.4. Relevant Experimental Data Collection. The experienced doctors examined cardiac ultrasound images of all the patients to record their cardiac function indicators. The subjects in the control group were tested on the day of physical examination, while patients with sepsis were tested on the $7^{\text {th }}$ day of admission.

$5 \mathrm{~mL}$ of venous blood was extracted on an empty stomach from all the subjects investigated, and it was centrifuged at $35,000 \mathrm{rpm}$ for 10 minutes. The products obtained from the centrifugation were detected by cardiac 
TABLE 1: Score sheet of SOFA.

\begin{tabular}{|c|c|c|c|c|c|c|c|}
\hline System & Examination program & 0 & 1 & 2 & 3 & 4 & Score \\
\hline \multirow{2}{*}{ Respiratory function } & $\mathrm{PaO}_{2} / \mathrm{FiO}_{2}(\mathrm{kPa})$ & $>53.33$ & $40-53.33$ & $26.67-40$ & $13.33-26.67$ & $<13.33$ & \\
\hline & Respiratory support (yes or no) & & - & - & Yes & Yes & \\
\hline \multirow{2}{*}{$\begin{array}{l}\text { Coagulation function } \\
\text { Liver function }\end{array}$} & Platelet $(109 / \mathrm{L})$ & $>150$ & $101-150$ & $51-100$ & $21-50$ & $<21$ & \\
\hline & Bilirubin (umol/L) & $<20$ & $20-32$ & $33-101$ & $102-204$ & $>204$ & \\
\hline \multirow{5}{*}{ Circulatory function } & Mean arterial pressure $(\mathrm{mmHg})$ & $>70$ & $<70$ & - & - & - & \\
\hline & Dopamine dose (uh/kg/min) & - & - & $\leq 5$ & $>5$ & $>15$ & \\
\hline & Adrenalin dose (ug/kg/min) & - & - & - & $\leq 0.1$ & $>0.1$ & \\
\hline & Norepinephrine dose (ug/kg/min) & - & - & - & $\leq 0.1$ & $>0.1$ & \\
\hline & Dobutamin (yes or no) & - & - & Yes & - & - & \\
\hline Neurological function & GCS scoring & 15 & $13-14$ & $10-12$ & $6-9$ & $<6$ & \\
\hline \multirow{2}{*}{ Renal function } & Creatinine (umol/L) & $<110$ & $110-170$ & $171-299$ & $300-400$ & $>400$ & \\
\hline & 24-hour urine output $(\mathrm{mL} / 24 \mathrm{~h})$ & - & - & - & $201-500$ & $<200$ & \\
\hline
\end{tabular}

troponin I (cTnI), N-terminal pro-B-type natriuretic peptide (NT-proBNP), H-FABP, and GPBB.

2.5. Statistical Analysis. SPSS 19.0 software was employed to analyze the data statistically. The enumeration results were expressed as mean \pm standard deviation, and the $t$-test was used for comparison between two groups. $P<0.05$ meant the difference was statistically significant.

\section{Results}

3.1. Scale-Invariant Feature Transform Algorithm Matching Results and Comparison. The first frame and the second frame of the image were taken as an example, and the following were the matching images obtained by sequential matching, reverse matching, and template matching, respectively (Figures 3-5). In the template matching, the one with more feature points in the two frames was used as a template for matching.

Figure 6 reveals that the results obtained by SIFT matching were further summarized. It suggested that there was little difference between the logarithm of matching points corresponding to the sequential matching and reverse matching methods, while the logarithm corresponding to the template matching method was greatly higher with better matching results.

\subsection{Comparison of General Data of Subjects in Each Group.} The comparison of general data among the subjects in the three groups is shown in Figure 7. It showed that there were no statistically remarkable differences in gender and age among all subjects in the three groups $(P>0.05)$. However, there were statistically substantial differences in SOFA scores among all subjects in the three groups $(P<0.05)$ and all subjects of the three groups had different hospital admission times with statistical meanings $(P<0.05)$.

3.3. Comparison of Experimental Results of Subjects in Each Group. Sepsis patients were given the same laboratory tests on the seventh day of admission, and the results of subjects in the three groups were compared as shown in Figure 8. Compared with the control group, the number of white blood cells (WBC), procalcitonin (PCT), NT-proBNP, cTnI, CK-MB, H-FABP, and GPBB were dramatically higher in groups $\mathrm{A}$ and $\mathrm{B}$, showing statistically substantial differences $(P<0.05)$. In addition, those values were the highest in sepsis patients of group B $(P<0.05)$.

3.4. Typical Cardiac Ultrasonic Images of Patients in Each Group. The typical cardiac ultrasonic images of subjects in the three groups were taken as shown in Figure 9. Figure 9(a) shows the cardiac ultrasonic image of one healthy person, the whole heart and left ventricle were normal, movement and morphological structure of the ventricular wall were not hugely changed, and the mitral valve was normal. Figure 9(b) shows the cardiac ultrasonic image of one septic patient with sound cardiac function; there was a normal diameter of the ventricular and a normal thickness of his or her heart wall; asymmetric cardiac hypertrophy did not emerge; and the ventricular wall motion amplitude was also basically regular. Besides, the cardiac ultrasonic image of one septic patient with cardiac insufficiency is shown in Figure 9(c), and the ventricular wall was extremely thickened and the aortic valve was obviously narrowed, so the left ventricle was considerably thickened.

3.5. Comparison of Cardiac Ultrasonic Data of Patients in Each Group. On the seventh day after admission, patients with sepsis underwent the same cardiac ultrasound examination as the subjects in the control group, and Figures 10 and 11 indicate the comparison of the results of subjects in the three groups. The values of left ventricular systolic function indicators (EDV, end-systolic volume (ESV), SV, CO, and E-point of septal separation (EPSS)) in groups A and B were extremely higher than the values of the control group with the statistically obvious differences $(P<0.05)$, and those values of subjects in group $B$ were the highest $(P<0.05)$. There were statistically substantial differences in the values of left ventricular diastolic function indicators (early transmitral flow velocity (E), late diastolic blood flow peak velocity (A), IVRT, E-peak deceleration time (EDT), systole $(\mathrm{S})$, and diastole (D)) of subjects from the three groups $(P<0.05)$, among which those values of patients in the control group were the highest $(P<0.05)$. 

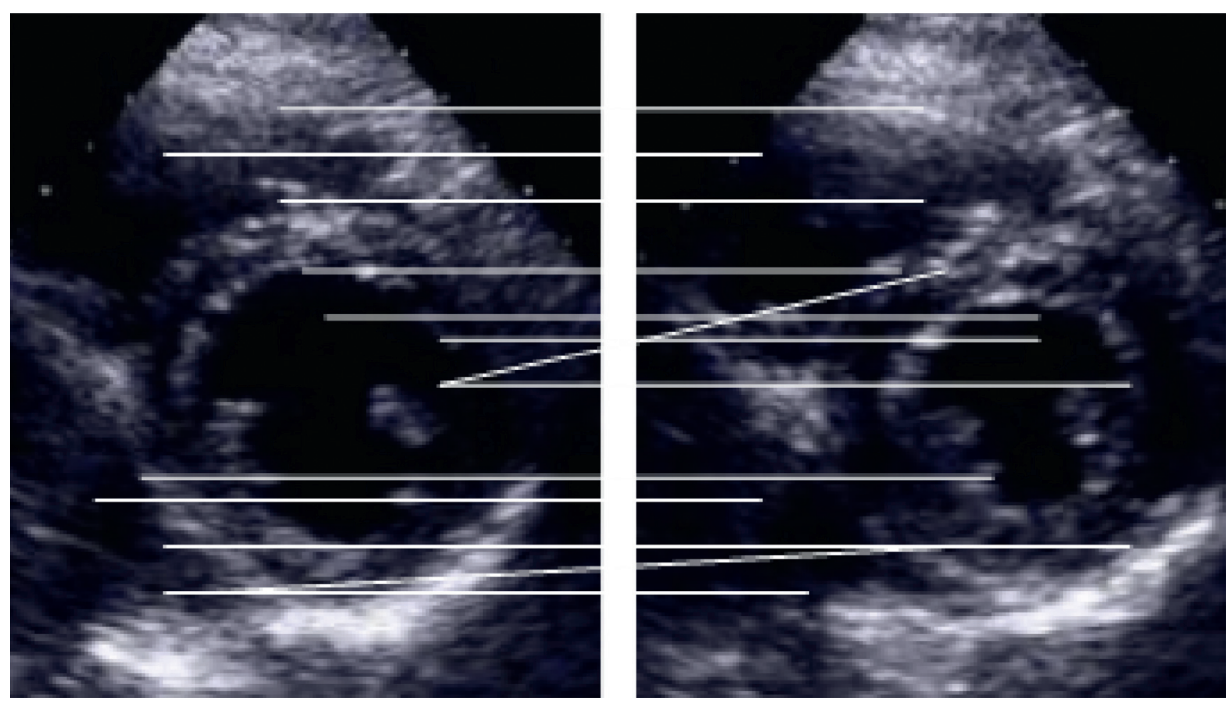

Figure 3: The result of sequential matching.
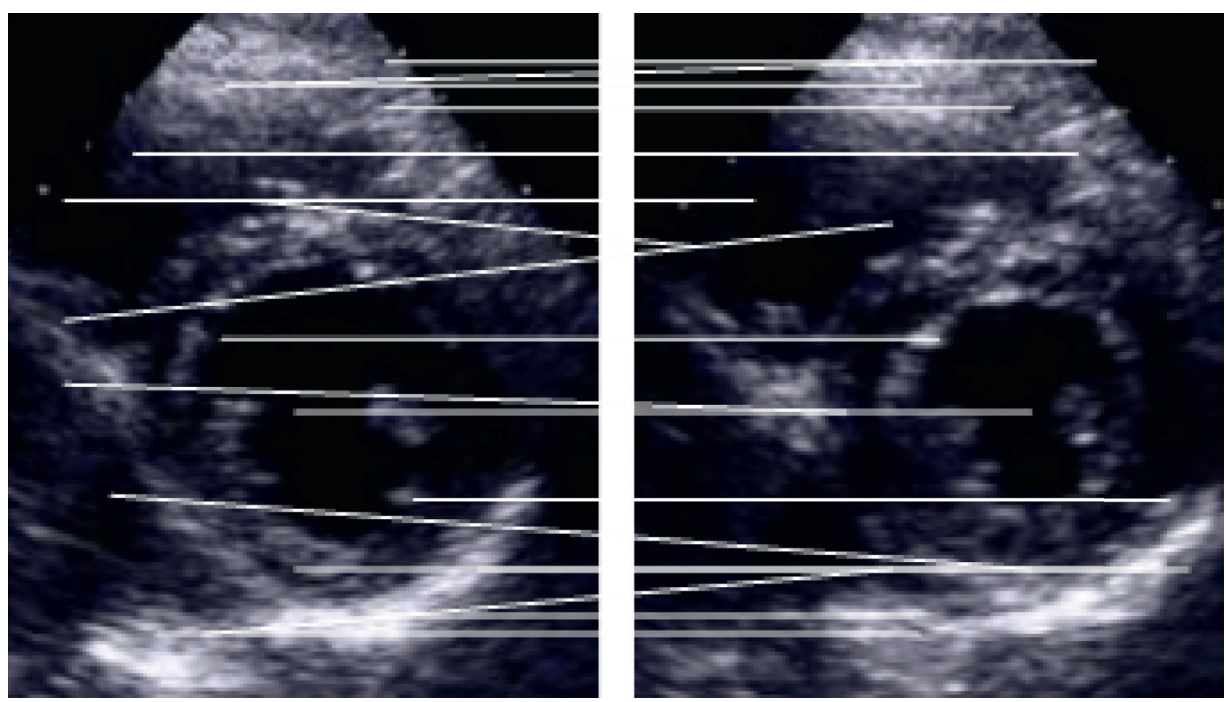

FIgURE 4: The result of reverse matching.
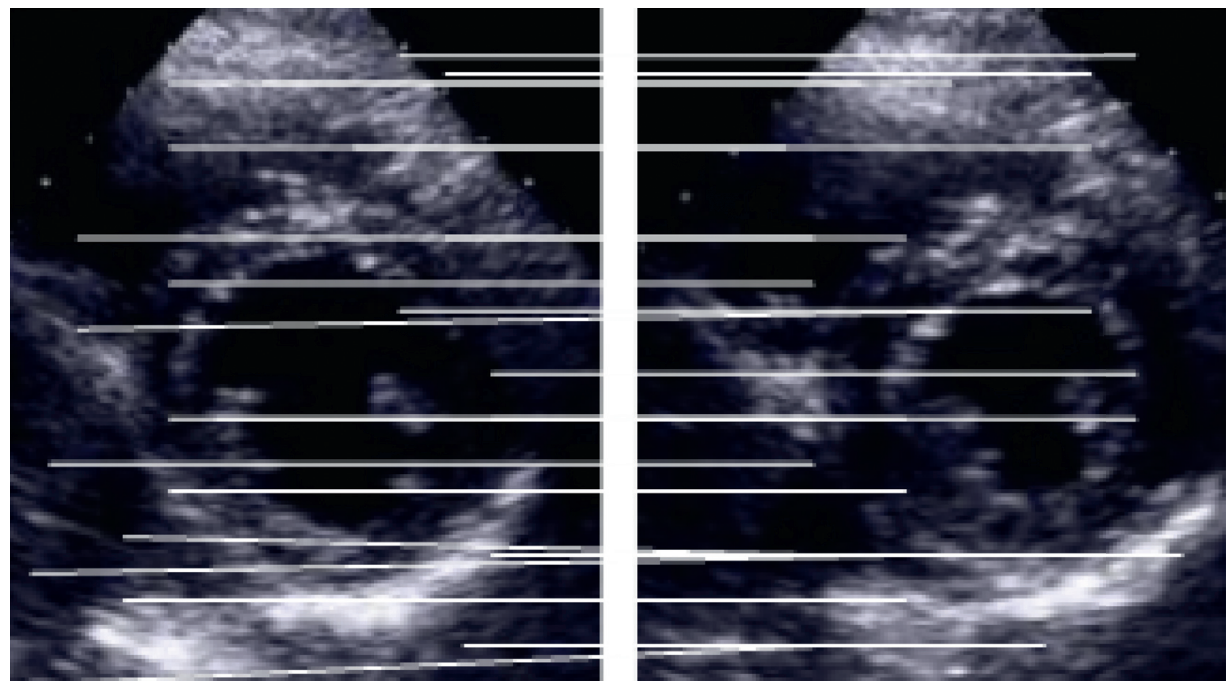

FIgURE 5: The result of template matching. 


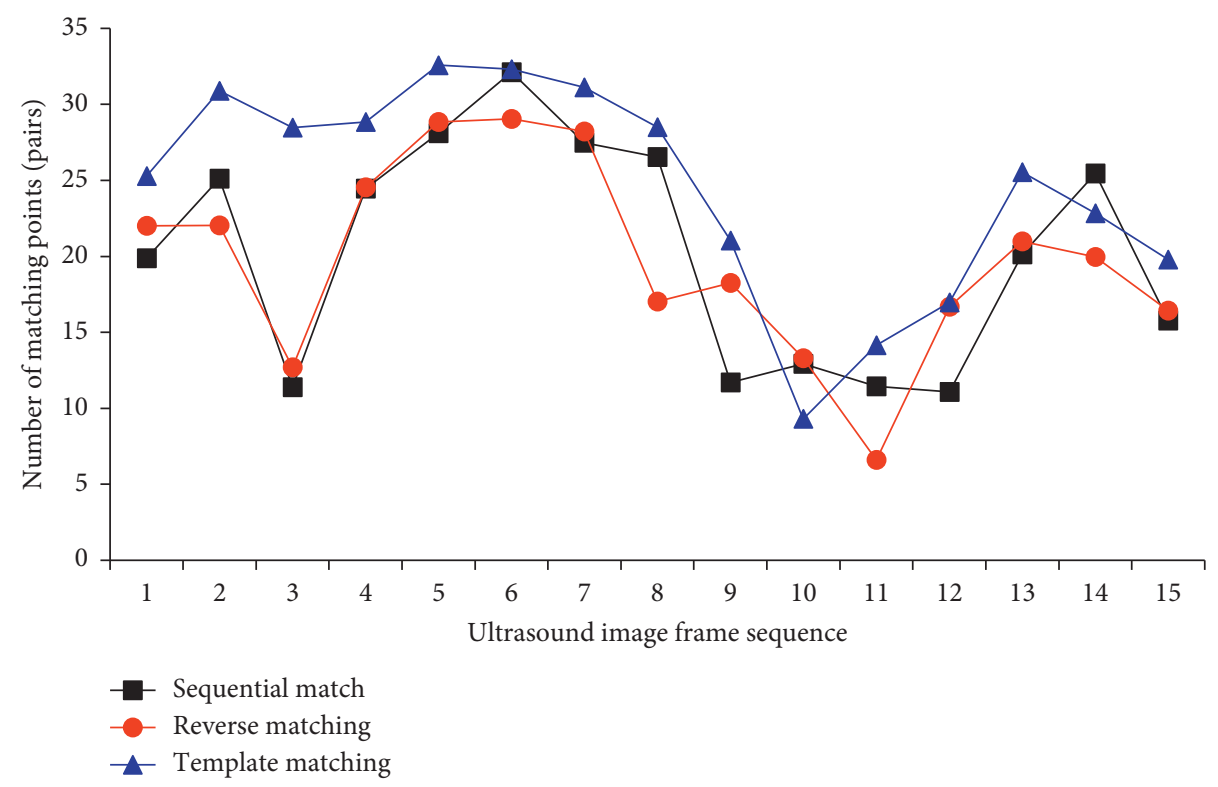

FIGURE 6: Comparison of the matching results of different methods.
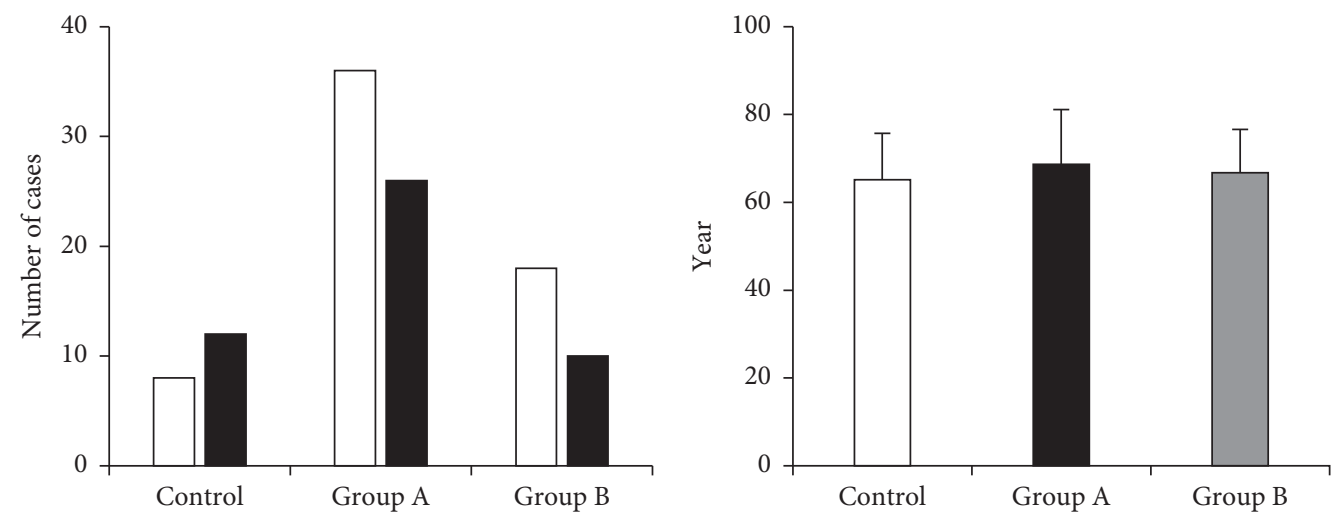

$\square$ Male

(a)

(b)

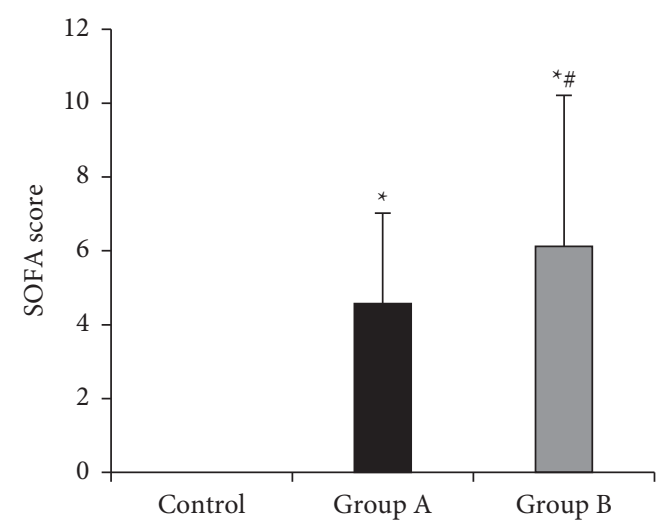

(c)

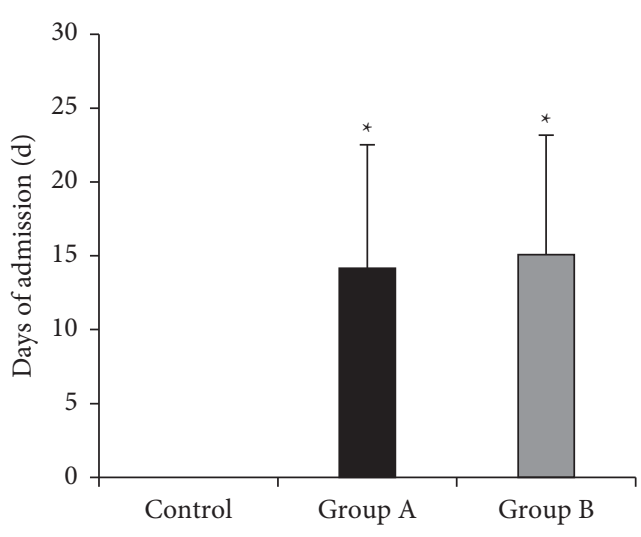

(d)

FIgURe 7: Comparison of general data among all subjects in the three groups. (a-d) Comparisons of gender, age, SOFA scores, and hospital admission time among subjects in each group, respectively; ${ }^{*}$ indicates that there was a statistically great difference compared with the control group $(P<0.05)$; and \# indicates the differences were statistically obvious compared to group A $(P<0.05)$. 


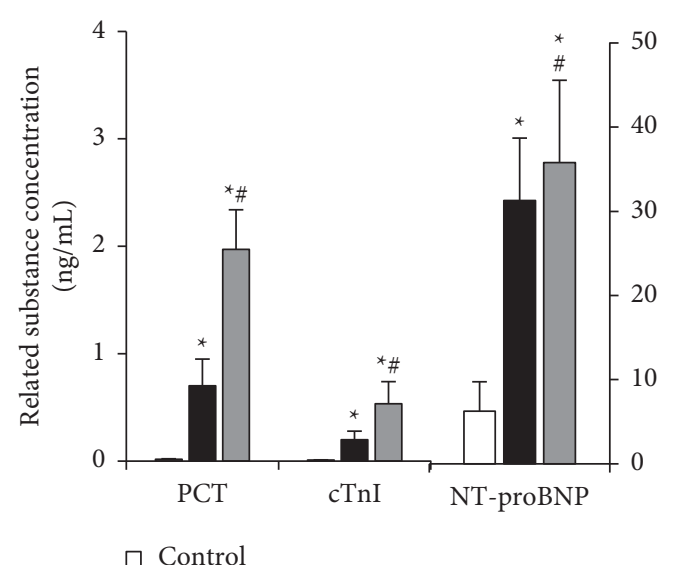

$\square$ Control

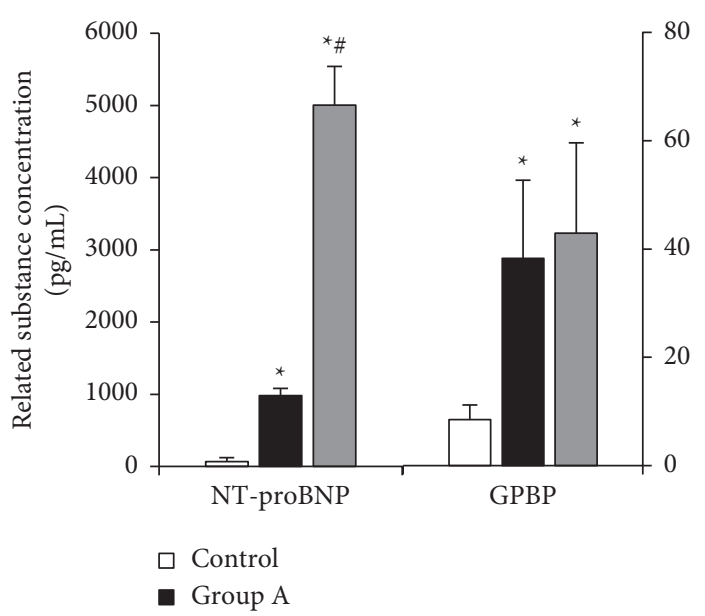

(b)

(a)

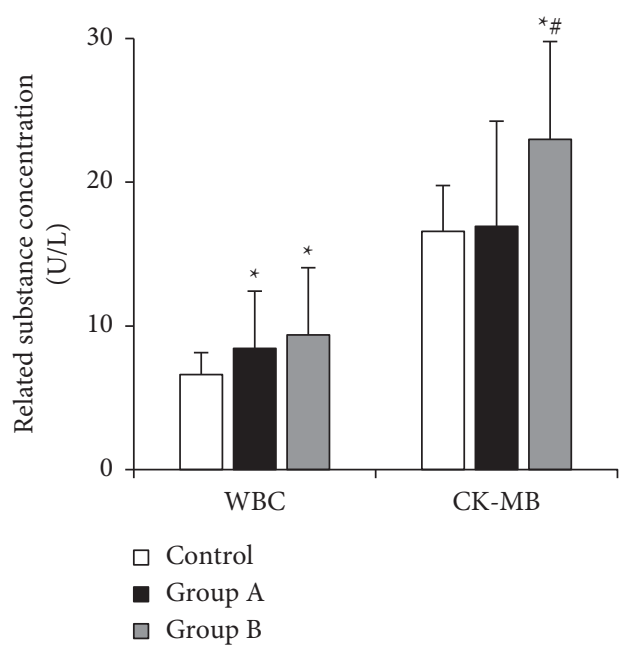

(c)

FIGURE 8: Comparison of experimental data among all subjects in the three groups. (a) Comparison on PCT, cTnI, and H-FABP among subjects in each group; (b) comparison on NT-proBNP and GPBP among subjects in the three groups; (c) comparison on WBC and CK-MB among subjects in each group; ${ }^{*}$ means that there was a statistically huge difference in contrast to the control group $(P<0.05)$, and \# indicates that the differences were statistically remarkable compared to group A $(P<0.05)$.

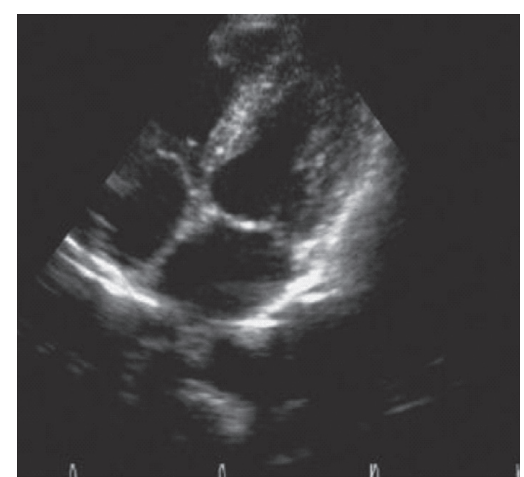

(a)

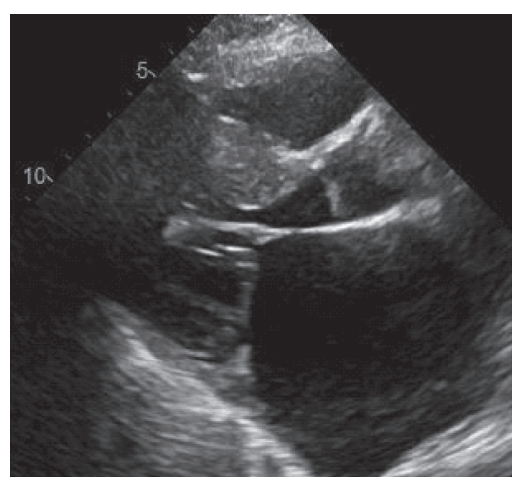

(b)

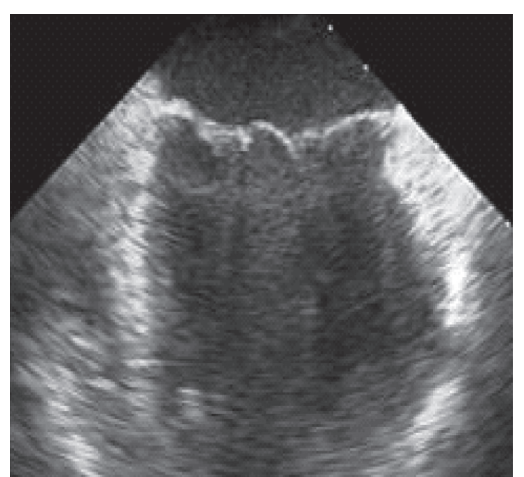

(c)

Figure 9: The typical cardiac ultrasonic images of subjects in each group. (a) The image of a healthy person, (b) the image of one septic patient with sound cardiac function, and (c) the image of one septic patient with cardiac insufficiency. 


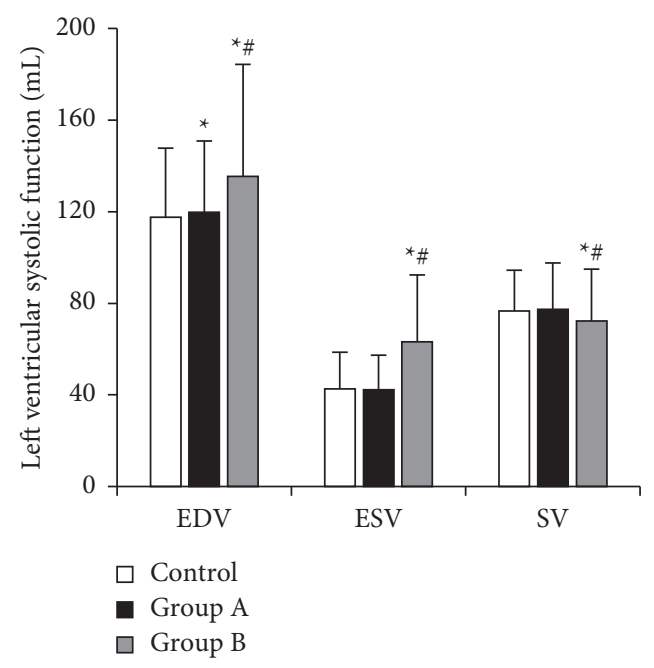

(a)

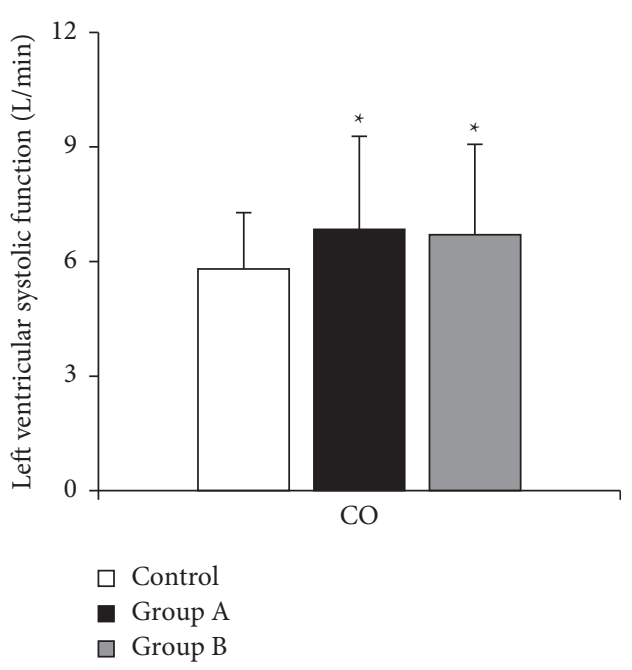

(b)

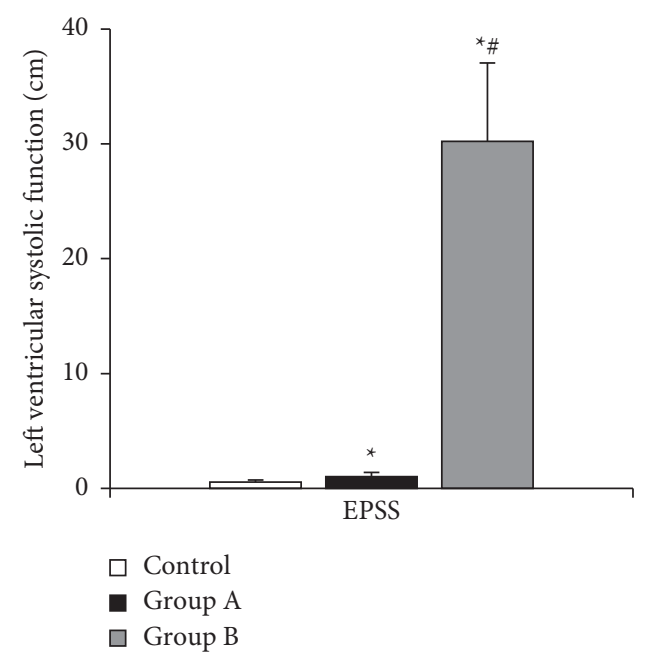

(c)

FIGURE 10: Comparison of left ventricular systolic function data of subjects in the three groups. (a) The comparison on EDV, ESV, and SV of subjects in the three groups; (b) the comparison on CO of subjects in each group; (c) the comparison on EPSS of subjects in the three groups; * reveals that the differences were statistically remarkable by comparing with the control group $(P<0.05)$; \# indicates that there was a statistically obvious difference in contrast to group A $(P<0.05)$.

\section{Discussion}

Sepsis refers to a series of uncontrolled conditions caused by inflammation in the body due to infection. When these conditions are serious, they often endanger the functions of living organs, so that the functions of related organs in the body could be reduced or even fail. On this basis, the definition of sepsis puts more emphasis on its role in organ function. Among these organ function changes, the cardiovascular system has been most affected with the most serious problems. Meanwhile, more personalized treatment is often required for sepsis accompanied by cardiovascular system damage. However, accurate judgment is often the premise and basis of personalized treatment. At present, the methods of studying myocardial changes have been developed from traditional tissue Doppler to angle-free speckle tracking with the improvement of various technologies
[14-17]. In this study, SIFT was applied to match cardiac ultrasonic images with three methods (sequential matching method, reverse matching method, and template matching method) to obtain the corresponding results, showing that the template matching method was better for image processing. Martin et al. [18] also confirmed in their study of cardiac ultrasound that there was little difference between sequential matching and reverse matching in cardiac ultrasonic processing, but there was room for improvement. Cardiac ultrasound is now the most important evaluation method for patients' cardiac function. Chen et al. [19] revealed in the study that patients with sepsis could be defined as having sepsis cardiac insufficiency when their EF values were less than $50 \%$. This study suggested that there were statistically obvious differences in cardiac ultrasonic indicators among subjects in the control group, group A, and group B. Kawaguchi et al. [20] found in a study of 262 


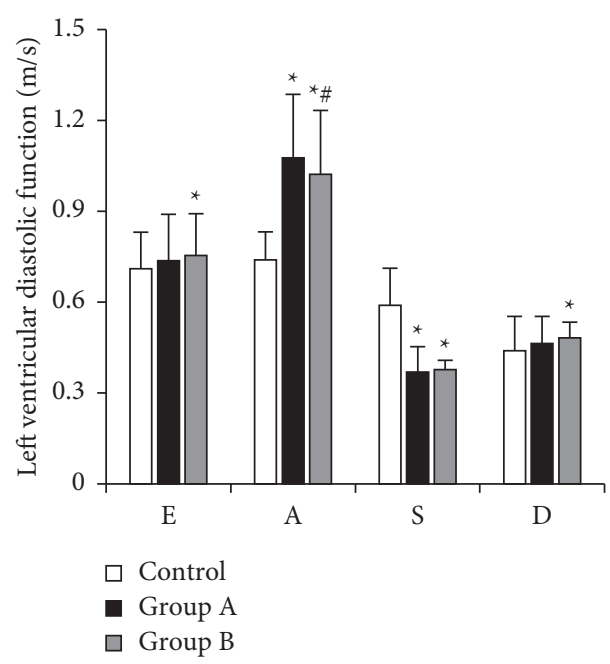

(a)

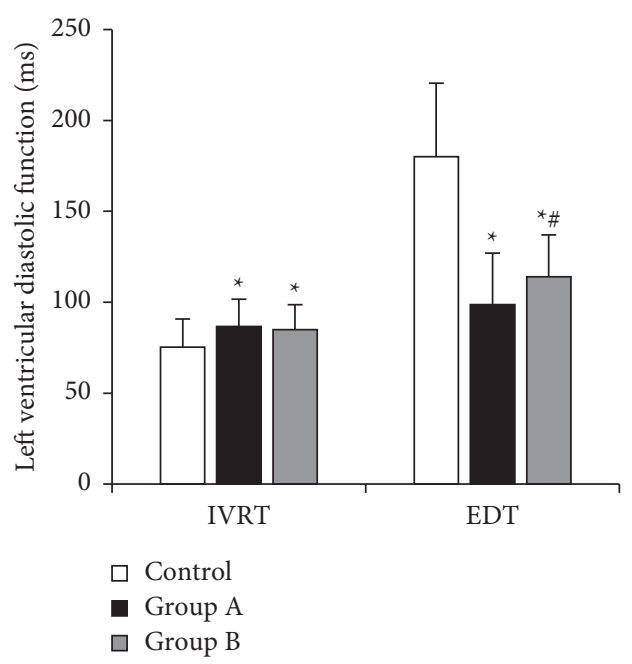

(b)

Figure 11: Comparison on left ventricular diastolic function data of subjects in each group. (a) The comparison on E, A, S, and D of subjects in the three groups; (b) the comparison on IVRT and EDT of subjects in each group; ${ }^{*}$ shows that there was a statistically great difference compared to the control group $(P<0.05)$, and \# indicates the differences were statistically considerable compared with group A $(P<0.05)$.

patients with severe sepsis that nearly half of the patients had left ventricular diastolic function impairment, $8.7 \%$ of the patients had simple systolic dysfunction, and $15.1 \%$ had both systolic and diastolic dysfunction. To a large extent, it disclosed that there were certain universalities of heart function problems in patients with sepsis.

The results of this study showed that the SOFA score of patients in group B was generally higher at admission, suggesting that sepsis patients with more severe single organ dysfunction or multiple organ dysfunction were more likely to have cardiac insufficiency at admission. However, this research result was not consistent with the results of domestic and foreign research data summarized by Strand et al. [21]. It was speculated that more complex standards were applied in this experiment while $\mathrm{EF}<50 \%$ was often used for diagnosis in current studies. This study also indicated that NT-proBNP was associated with whether patients suffered from cardiac insufficiency or not. NT-proBNP is a common cardiac function evaluation marker with high sensitivity and specificity at present. However, if patients with sepsis at admission received a large amount of fluid resuscitation, it would often lead to an increase in NT-proBNP due to iatrogenic cardiac volume load, which has been confirmed in the study of Gao et al. [22].

\section{Conclusion}

In this study, the SIFT operator of STA was studied to get the corresponding verification. The ultrasonic images and physiological characteristics of sepsis patients with cardiac insufficiency were further studied, and the results revealed that there were changes in cardiac imaging of patients in group $B$ on the $7^{\text {th }}$ day after the onset, and the values of $\mathrm{H}-\mathrm{FABP}, \mathrm{NT}$-proBNP, and $\mathrm{A}^{\prime}$ were all changed in contrast to the control group and group $B$, which could be regarded as predictors of sepsis cardiac insufficiency. The algorithm in the study was only limited to cardiac ultrasonic images, and it should be further explored whether STA could also play a role in other ultrasonic images. Moreover, there were few samples in this study, and the sources of sepsis patients were not subdivided, which needed further in-depth study. The results of this study could provide a reference for improving the efficiency of the clinical diagnosis of sepsis.

\section{Data Availability}

The data used to support the findings of this study are available from the corresponding author upon request.

\section{Conflicts of Interest}

The authors declare no conflicts of interest.

\section{References}

[1] S. Vallabhajosyula, J. C. Jentzer, J. B. Geske et al., "New-onset heart failure and mortality in hospital survivors of sepsisrelated left ventricular dysfunction," Shock, vol. 49, no. 2, pp. 144-149, 2018.

[2] G. Abou Dagher, K. Hajjar, C. Khoury et al., "Outcomes of patients with systolic heart failure presenting with sepsis to the emergency department of a tertiary hospital: a retrospective chart review study from Lebanon," BMJ Open, vol. 8, no. 7, Article ID e022185, 2018.

[3] A. M. N. Walker, M. Drozd, M. Hall et al., "Prevalence and predictors of sepsis death in patients with chronic heart failure and reduced left ventricular ejection fraction," Journal of American Heart Association, vol. 7, no. 20, Article ID e009684, 2018.

[4] C.-w. Lin, W. Tang, F. Wen, J.-j. Chen, X.-l. Zeng, and Z.-g. Chen, "Diagnostic accuracy of NT-ProBNP for heart failure with sepsis in patients younger than 18 years," PLoS One, vol. 11, no. 1, Article ID e0147930, 2016. 
[5] R. A. Khan, N. A. Khan, S. R. Bauer et al., "Association between volume of fluid resuscitation and intubation in highrisk patients with sepsis, heart failure, end-stage renal disease, and cirrhosis," Chest, vol. 157, no. 2, pp. 286-292, 2020.

[6] X. Wang, L. Su, R. Yang, H. Zhang, and D. Liu, "Myocardial strain/stress changes identified by echocardiography may reveal early sepsis-induced myocardial dysfunction," Journal of International Medical Research, vol. 46, no. 4, pp. 14391454, 2018.

[7] J. Li, B. Ning, Y. Wang et al., "The prognostic value of left ventricular systolic function and cardiac biomarkers in pediatric severe sepsis," Medicine, vol. 98, no. 13, Article ID e15070, 2019.

[8] S. Vallabhajosyula, H. A. Rayes, A. Sakhuja, M. H. Murad, J. B. Geske, and J. C. Jentzer, "Global longitudinal strain using speckle-tracking echocardiography as a mortality predictor in sepsis: a systematic review," Journal of Intensive Care Medicine, vol. 34, no. 2, pp. 87-93, 2019.

[9] A. A. Havaldar, "Evaluation of sepsis induced cardiac dysfunction as a predictor of mortality," Cardiovascular Ultrasound, vol. 16, no. 1, p. 31, 2018.

[10] F. Sanfilippo, C. Corredor, N. Fletcher et al., "Left ventricular systolic function evaluated by strain echocardiography and relationship with mortality in patients with severe sepsis or septic shock: a systematic review and meta-analysis," Critical Care, vol. 22, no. 1, 2018.

[11] M. Balik, V. Matousek, M. Maly, and T. Brozek, "Management of arrhythmia in sepsis and septic shock," Anaesthesiology Intensive Therapy, vol. 49, no. 5, pp. 419-429, 2017.

[12] M. Feng, J. I. McSparron, D. T. Kien et al., "Transthoracic echocardiography and mortality in sepsis: analysis of the MIMIC-III database," Intensive Care Medicine, vol. 44, no. 6, pp. 884-892, 2018.

[13] T. P. Shiji, S. Remya, and V. Thomas, "Computer aided segmentation of breast ultrasound images using scale invariant feature transform (sift) and bag of features," Procedia Computer Science, vol. 115, pp. 518-525, 2017.

[14] B. Haileselassie, E. Su, I. Pozios, F. Teresa, T. Reid, and A. Theodore, "Strain echocardiography parameters correlate with disease severity in children and infants with sepsis," Pediatric Critical Care Medicine, vol. 17, no. 5, pp. 383-390, 2016.

[15] H. S. Jeong, T. H. Lee, C. H. Bang, K. Jong-Ho, and J. H. Soon, "Risk factors and outcomes of sepsis-induced myocardial dysfunction and stress-induced cardiomyopathy in sepsis or septic shock: a comparative retrospective study," Medicine (Baltimore), vol. 97, no. 13, Article ID e0263, 2018.

[16] L. Martin, M. Derwall, C. Thiemermann, and T. Schurholz, "Herz in der sepsis: molekulare mechanismen, diagnose und therapie der septischen kardiomyopathie [heart in sepsis: molecular mechanisms, diagnosis and therapy of septic cardiomyopathy]," Anaesthesist, Der, vol. 66, no. 7, pp. 479-490, 2017.

[17] K. Pilarczyk, J. Renner, and N. Haake, "Akutes rechtsherzversagen auf der intensivstation: pathophysiologie, monitoring, therapie [acute right heart failure on the intensive care unit:pathophysiology, monitoring and management]," Medizinische Klinik - Intensivmedizin und Notfallmedizin, vol. 114, no. 6, pp. 567-588, 2019.

[18] L. Martin, M. Derwall, S. Al Zoubi et al., "The septic heart: current understanding of molecular mechanisms and clinical implications," Chest, vol. 155, no. 2, pp. 427-437, 2019.
[19] J. Chen, B. Wang, J. Lai et al., "Trimetazidine attenuates cardiac dysfunction in endotoxemia and sepsis by promoting neutrophil migration," Frontiers in Immunology, vol. 9, 2018.

[20] S. Kawaguchi, M. Okada, E. Ijiri, and D. Koga, “ $\beta_{3}$-Adrenergic receptor blockade reduces mortality in endotoxin-induced heart failure by suppressing induced nitric oxide synthase and saving cardiac metabolism," American Journal of Physiology Heart and Circulatory Physiology, vol. 318, no. 2, pp. H283H294, 2020.

[21] M. E. Strand, J. M. Aronsen, B. Braathen et al., "Shedding of syndecan-4 promotes immune cell recruitment and mitigates cardiac dysfunction after lipopolysaccharide challenge in mice," Journal of Molecular and Cellular Cardiology, vol. 88, pp. 133-144, 2015.

[22] M. Gao, X. Wang, X. Zhang et al., "Attenuation of cardiac dysfunction in polymicrobial sepsis by MicroRNA-146a is mediated via targeting of IRAK1 and TRAF6 expression," The Journal of Immunology, vol. 195, no. 2, pp. 672-682, 2015. 